\title{
Design of Diagnosis and Monitoring System of Heart Related Diseases using Fuzzy Inference System
}

\author{
Lokanath Sarangi \\ ITER, Siksha 'O' Anusandhan \\ University \\ Bhubaneswar, Odisha, India \\ lokanathsarangi@yahoo.com
}

\author{
Mihir Narayan Mohanty \\ ITER, Siksha 'O' Anusandhan \\ University \\ Bhubaneswar, Odisha, India \\ mihir.n.mohanty@gmail.com
}

\author{
Srikanta Pattnaik \\ ITER, Siksha 'O' Anusandhan \\ University \\ Bhubaneswar, Odisha, India \\ srikantapatnaik@soauniversity.ac.in
}

\begin{abstract}
Human desire increases day-by-day in different aspects. It requires the technology to be compatible accordingly. Fast and user friendly access are major challenges for different applications. One of the applications is hospital management for patient care, diagnosis and treatment. Modern health care provide different type of provision for people using electronic media. Recently research explores the cardiac diagnosis and care using modern equipment and facilities. In this paper an approach has been considered to diagnose heart diseases in an intelligent manner. The model is designed using fuzzy logic in which the rule based principle is applied to satisfy the objective. Keeping view on multi agent system the model is developed. The diagnosis of the patient is performed using Fuzzy Inference System (FIS). Once the pathological test results are obtained these will help to form the rules of the model and it works for the diagnosis in convenient way. Further the result of detection is communicated through internet or SMS for monitoring and post care purpose. The simulated result shows its performance that can be helpful to the physicians as well as the patients from distant places.
\end{abstract}

Keywords-Multi Agent System, Fuzzy Inference System, Diagnosis, Fuzzy Rule Base

\section{INTRODUCTION}

Intelligent techniques are applied in many application areas to improve the reliability and smart service. One of the most important applications is health care system. Researchers are trying to improve these units to provide better service to patients. The outcomes can support the physicians also in a better way in comparison to the previous methods. It tends towards multi agent system concept and its research has been spread over most of the areas such as Computer science, Engineering, Medicine, and business organizations etc. The system comprises with a set of models based on the knowledge base of related field .These models may be reaching and revised. Though the objective of multi agent system is for compression and co-ordination between agents it requires refinement according to the desire of the client agents. The system has four types of features like
1. Communication among agents

2. Autonomy

3. Agent behavior

4. Heterogeneity.

Each feature can be developed through software modules for particular environment. To form the system agents are the software design paradigms based on the environment. The authors have tried to develop the module of diagnosis and monitoring subsystems of e-health care system.

Health consciousness has expanded the life span of the human being. To improve the quality of life in the society smart health service is indispensable. E-health is the substantial way to fast service. By this technology we can address and collect a database of the most common chronic diseases and their complications which could potentially help to elucidating the main reasons and reducing the risk of their occurrence. E healthcare plays a vital role in this area. By virtue of this quality health service can be provided in rural areas which are far from reach of the medical facility. Also the home monitoring of health care can save both time as well as the expenses of the patient [1]. E-Health technologies have a significant future impact on patient for empowerment in health maintenance and decision making supporting self-care.

The healthcare units consists of several factors and some of the agents as the healthcare communicate in smart way and also provide support to different agents like patient, patho-care, physicians, hospitals and also to rural areas. It is termed as e-health. It is inter changeable with health information in most of the cases these are useful to store the health records support for telemedicine and services to the consumers. Due to the population in rural area, are more than the urban area the e-health service can be useful for the patients and practitioners. The system may take the help of 
wireless communication or internet or mobile based system. So another issue is there of multiple health records of the patient in different health care units based on their problems. It is necessary to access that information from different sources and to manage the flow for treatment. So for this treatment also the system may use the e-prescription and suggestions to the patient of different places. So it became a great challenge for researchers with insufficient infrastructure. So this is helpful to the patients and can be allotted to the critical patients. To detect and monitor cardiac disease in intelligent way is a great challenge in the present scenario. Establishment of co-ordination among health care units such as patient, physician, health records, diagnostic centers etc. can disburse the service effectively.

A complex system can be understood and managed in a better way by modeling and simulation. An agent is a single entity having knowledge, objective and capability of carrying out certain task. An interoperable self- independent co-operative multi agent system can address the complex environment involved in healthcare [2]-[6]. In the field of computational intelligence design Fuzzy Logic has proved itself as a powerful tool for developing an intelligent knowledge base system in healthcare. Various bio sensors fitted to the body of the patient provide physical parameters to the fuzzy logic controller. The fuzzification of these data is transformed into fuzzy variables. These fuzzy variables are easy to understand and its modeling helped to reduce the human error in prediction [6] [7].

Communication is an inevitable tool in this system. Transfer of health record without loosing it's originality a great challenge. Researchers have tried for communication among all the agents using various techniques [6]-[10]. The research on this field encouraged us to implement fuzzy logic for detection of the cardiac disease and the information regarding the communication is established with the help of a microcontroller based module.

In this work we have considered one of the chronic diseases as the cardiac problem that may occur due to aging effect. In section 2 some of the related works have been cited and the proposed methodology is explained in section 3 . The possible outcome is shown in section 4 and section 5 concludes this piece of work.

Though different approaches have been made since a long period, still there is a gap of implementation and accuracy. Based on the literature our proposed work is explained in the following section.

\section{RELATED LITERATURE}

For the development of e-health care system, researchers have been tried since a long period. Some of them focused on multi-agent system whereas most of them analyze the diagnostic center. Attention has been given to the field of signal and image processing along with telemedicine system [2-4, 7-13]. This section provides the related work in this field. A little amount of works on this area has been approached by many authors. Some of those are cited in this section to review the literature.

In [11], authors have developed a processor for transmission of signals from a patient over a Bluetooth link to a mobile telephone. Various algorithms were introduced to reduce the number of rules, searches with high predictive accuracy for heart diseases. Models were proposed for feature selection of clinical data for detection of patients with CHD. Imaging technique was utilized to coronary angiography for predicting the risk factors of CHD. Particle swarm optimization (PSO) was used for optimization for better accuracy of $99.73 \%$. Adaptation and efficient utilization of suitable algorithm in development of effective healthcare systems has been a major challenge as the Information recovered from the wearable devices in e-health care system is complex in nature. Using suitable algorithm it can be represented to the physicians for decision and detection of diseases. Considerable inputs on development of suitable healthcare system including Electromechanical Wave Imaging (EWI) and Wearable Smartphone based platform for detection and diagnosis of critical diseases has been investigated by different authors [12]-[13].Detection and proper identification of diseases desires the selection of efficient classification methods. Among different classifiers, Relative Associated Density (RAD) method, Artificial Neural Network (ANN) and Decision Tree Method etc. has been quite effective in this direction. ANN is a class of classifier that resembles the biological neurons of human being and can describe complex input and output relations efficiently. Few of the classifiers such as RAD, ANN, k-NN ( $\mathrm{k}$ nearest neighbor) and decision tree has been utilized to detect the cardiac diseases successfully, has been the major influence in the field of health care [14]-[16]. The authors have attempted for cost-sensitive $\mathrm{k}-\mathrm{NN}$ for the patients subjected to high-risk heart disease [16]. Features representing these signals received from the testing machines have to be robust and reliable so as to describe the symptoms of these diseases. Different feature extraction techniques and algorithms have been experimented for monitoring complicated health related issues. Use of software tools and suitable feature extraction methods dealing with testing and measurement for abnormal heart sounds has been investigated in [17]. Prediction algorithm that can detect these diseases is one of the major tasks. Efficient prediction algorithm that can suitably describe the symptoms of the diseases from the signals received during testing of the patients has been an area needs further exploration. In the work of Lakshmi et.al a comparison of different data mining techniques and application of android concept that can predict cardiac disease has been mentioned [18] [19]. Classifiers such as random forest classifier and support vector machine classifier (SVM) used to recognize heart beat signal to explore for Hypertrophic Cardiomyopathy Identification [20]. The application of Mobile Phone Based eHealth Monitoring, displaying of ECT signals, Continuous and intelligent monitoring of ECG signal and its' classification can provide potential inputs to medical persons and the patients has been the major work found in [21]. 
Similarly, details on E health care system using multi-agent platform and fuzzy-logic based healthcare system for smart delivery of health service has been proposed for the patient receiving treatment from their home [22-27].

\section{Proposed MulTi-AGENT SysteM}

For The proposed system is shown in Fig. 1. It consists of intelligent agents to detect the disease. Once the abnormalities are detected it will communicate to the physician as well as to the patient.

Next to it the different agents are defined that follows the intelligent detector using Fuzzy Logic.

Patient Agent- Patient who is suffering will communicate to the hospital registration with the help of a communicating device like mobile phone or PC.

Hospital Registration-The hospital registration will store the parameters in the server and forward the parameters to the doctor 1 agent for recommendation of pathological tests.

Doctor1- The doctor 1 agent acts like the OPD of the hospital. It uses the fuzzy If Then rules for suggesting the pathological tests required for the patient.

Diagnostic Centre Agent-The data generated from the patient is sent to the diagnostic agent for analysis and prediction of the disease. The server sends as well as receives the data from this unit.

Doctor 2 or Specialist Agent-This is communicated with doctor. It will send email/SMS to the doctor regarding the abnormality for getting the prescription. The prescription is generated and delivered to the patient. If the Doctor requires investigating any medical history of the patient or time to time variation of the parameters of the patient, he can be able to access the server for monitoring purpose.

Server Agent- Server serves the information to all. It sends the data for diagnosis to diagnostic centre and receives the report. Similarly the reports are sent to the physician. The decision of data flow and management of overall operations is done by it.

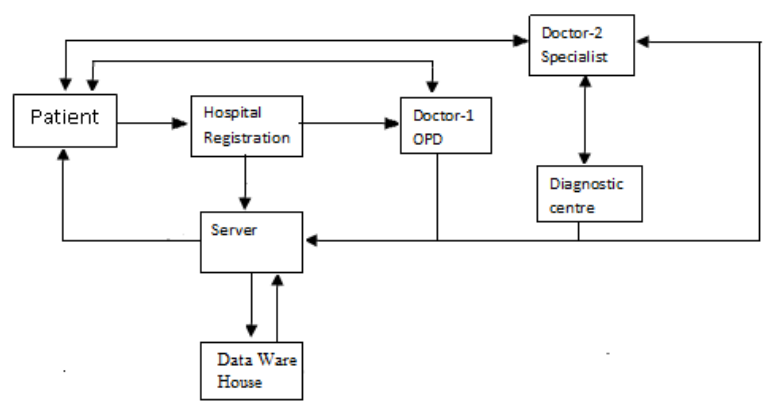

Fig. 1 Multi-agent Structure for Patient Consultancy Using Fuzzy System

Fig. 2 describes the data flow in diagnostic agent. The patient is advised to send his symptoms to the diagnostic center .Based on those data the diagnostic center analyze for prediction of the disease. In this work we have used fuzzy inference system for detection of heart related disease.

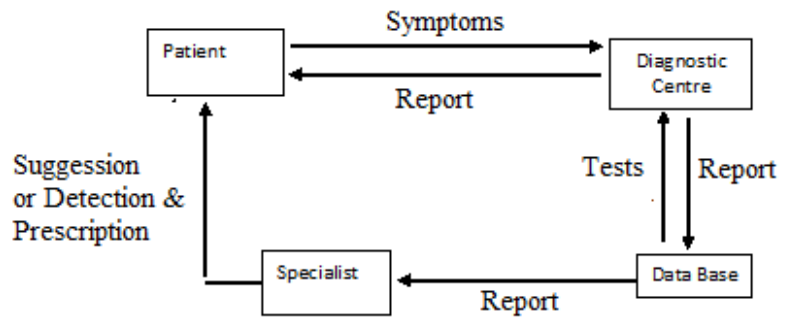

Fig.2 Diagnostic Centre Structure

Fig. 3 describes the agent based consultancy of the patient. The patient uploads his symptoms as well as his parameters to the server through hospital registration. The uploaded data are forwarded by the server for testing at the diagnostic center. If it is found that the condition of the patient is normal then an email or SMS is sent to the patient along with remedial steps to be taken by him depending upon his health condition.

If any abnormality is observed then the diagnostic center agent will inform the concerned specialist i.e. Doctor 2 for immediate action. Here the fuzzy IF THEN rules is implemented for providing information to the doctor. The doctor can check the reports of the patient by sending a request to the server.

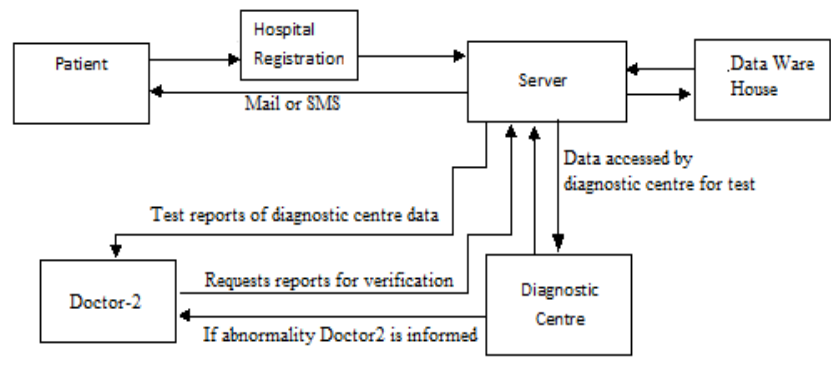

Fig. 3 Information Centre

\section{HEART DISEASE DIAGNOSIS USING FUZZY INTERFERENCE SYSTEM}

Rule based fuzzy system acts as the diagnosis center. The test and result are analyzed using if-then-elselogic.

The fuzzy set is defined as $A=\Sigma x i \in X \mu A(x i) / x i$, if $X$ is a collection of discrete objects, and $A=\int X \mu A(x) / x$, if $X$ is a continuous space [36].For the design of the system, triangular membership function is used and is explained as follows.

It is specified by three parameters those can be formulated for the specific problem.

Let the set of parameters are $\{a, b, c\}$

$$
\begin{gathered}
\text { Triangle }\left(x, x_{s}, b, c\right)=0 \text { if } x \leq a_{x} \\
=(x-a) /(b-a) \text { if } a \leq x \leq b ; \\
=(x-b) /(c-b) \text { if } b \leq x \leq c_{x} \\
=0 \text { if } c \leq x
\end{gathered}
$$

The rules can be implemented for the triangular membership function. If-then rule is of the form "If $\mathrm{x}$ is $\mathrm{A}$ then $\mathrm{y}$ is B" 
where A and B are linguistic values defined by fuzzy sets on universes of discourse $X$ and $Y_{x}$ respectively. " $x$ is $A$ " is called antecedent and " $y$ is $B$ " is called consequent.

The problem for diagnostic agent is formulated as follows.

For the adaptive cardiac disease detection using the fuzzy inference system, the following parameters are taken

- BP taken here in the range 90 to 190 (in $\mathrm{mm} \mathrm{Hg}$ )

- Serum Cholesterolin the range 120-560 (Mg/dl)

- $\operatorname{FBS} 110(\mathrm{Mg} / \mathrm{dl})$

- ECG ST-T wave abnormality

The above inputs are chosen for the following reason

- The function of heart is normal if BP is normal. If $\mathrm{BP}$ rises abnormally then this indicates the patient may suffer from cardiac disease. So BP is concerned with the heart disease detection.

- Deposition of TG narrows the blood circulation path. Excess of Cholesterol in blood gradually narrows the circulatory path and the patient is detected suffering from cardiac disease.

- Sugar is the silent killer. If exists beyond the normal value then there are chances of heart disease.

- ECG signal is the best predictor of heart disease. If ST-T wave abnormality is observed the though all the above tests show normal still then the patient is detected as cardiac

The fuzzy inference system that uses the above mentioned parameters, BP, FBS, Cholesterol and ECG ST-T abnormality, as its inputs, and Detection as its output. A schematic diagram of our system is shown in Fig.4. The linguistic variables used with fuzzy sets with different data such as ECG, BP, FBS etc. has been tabulated in Table I through Table IV.

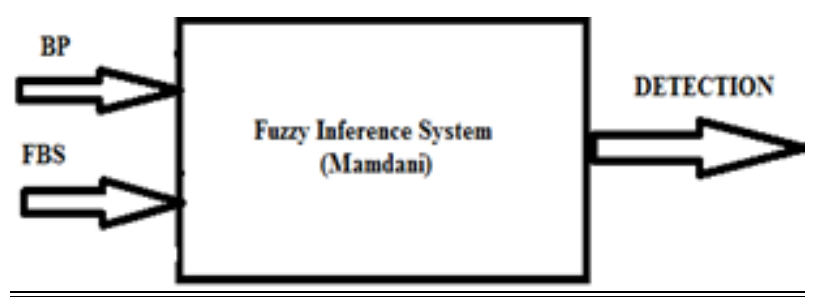

Fig. 4 schematic diagram of fuzzy inference system

TABLE I

LINGUISTIC VARIABLES VERSUS FUZZY SET FOR BP AND FBS

\begin{tabular}{|l|l|}
\hline Linguistic variable & Fuzzy set \\
\hline BP & $\{$ Low, Normal, High $\}$ \\
\hline FBS & $\{$ Low, Normal, High $\}$ \\
\hline Detection & $\{$ No, Yes $\}$ \\
\hline
\end{tabular}

The membership functions input BP Range

\begin{tabular}{ll}
\hline Low & {$[40 / 90-70 / 100]$} \\
Normal & {$[70 / 110-80 / 120]$} \\
High & {$[90 / 130$ and above $]$}
\end{tabular}

The membership functions input FBS Range

Low

Normal

[60-70]

High

[70-120]

[120 and above]
The fuzzy inference system that uses the above mentioned parameter, ECG, as its input, and Detection as its output. A schematic diagram of our system is shown in Fig. 5.

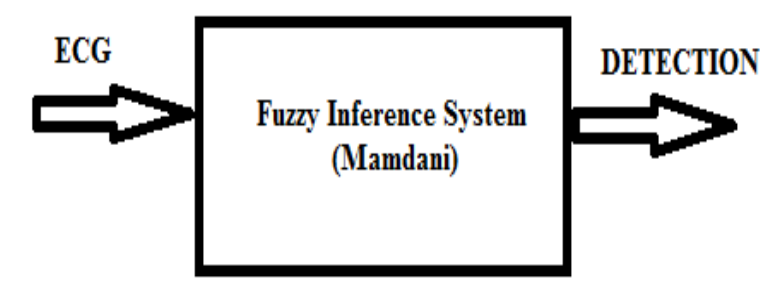

Fig. 5: schematic diagram of fuzzy inference system

TABLE II

LINGUISTIC VARIABLES VERSUS FUZZY SET FOR ECG

\begin{tabular}{|c|c|}
\hline Linguistic variable & Fuzzy set \\
\hline ECG & $\{$ Low, Normal, High $\}$ \\
\hline Detection & $\{$ No, Yes $\}$ \\
\hline
\end{tabular}

The membership functions input ECG $\underline{\text { Range }}$

Low

High

The fuzzy inference system that uses the above mentioned parameters, ECG,FBS, and BP as its inputs, and Detection as its output. A schematic diagram of our system is shown in Fig. 6.

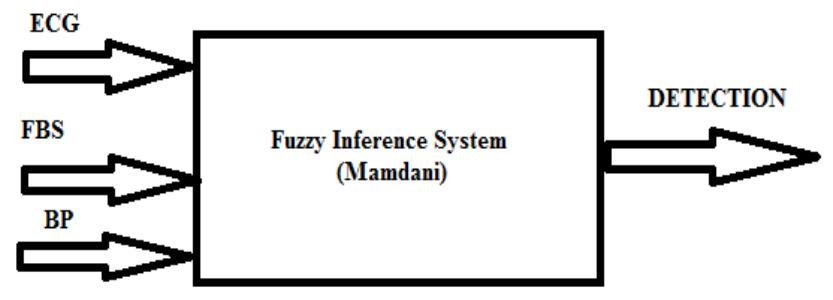

Fig. 6: schematic diagram of fuzzy inference system

TABLE II

LiNGUISTIC VARIABLES VERSUS FUZZY SET FOR ECG, BP AND FBS

\begin{tabular}{|c|c|}
\hline Linguistic variable & Fuzzy set \\
\hline ECG & $\{$ Normal, Abnormal $\}$ \\
\hline BP & $\{$ Low, Normal, High $\}$ \\
\hline FBS & $\{$ Low, Normal, High $\}$ \\
\hline Detection & $\{$ No, Yes $\}$ \\
\hline
\end{tabular}

The membership functions input ECG Range

Low

\section{0}

Normal0.5

Abnormal

1

The membership functions input BP Range

\begin{tabular}{ll}
\hline Low & {$[40 / 90-70 / 100]$} \\
Normal & {$[70 / 110-80 / 120]$} \\
High & {$[90 / 130$ and Above $]$}
\end{tabular}

The membership functions input FBS Range

\begin{tabular}{lc}
\hline Low & {$[60-70]$} \\
Normal & {$[70-120]$} \\
High & {$[120$ and above $]$}
\end{tabular}


The fuzzy inference system that uses the above mentioned parameters, ECG,FBS,BP and Cholesterolas its inputs, and Detection as its output. A schematic diagram of our system is shown in Fig. 7.The membership function of different data inputs such as BP, FBS, cholesterol and ECG has been graphically shown in Fig. 8 through Fig. 11.

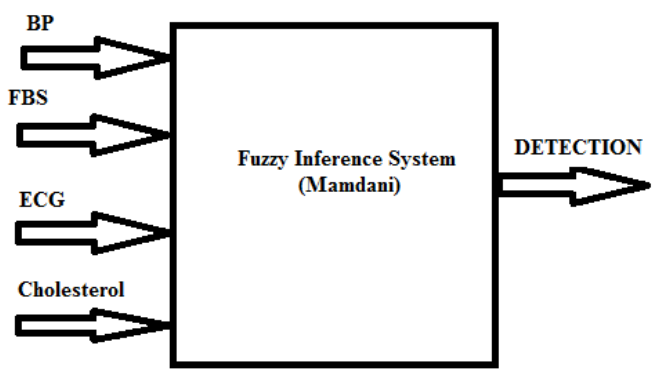

Fig. 7 Schematic diagram of fuzzy inference system

TABLE III

LINGUISTIC VARIABLES USED WITH FUZZY SET FOR ECG, BP AND FBS

\begin{tabular}{|c|c|}
\hline Linguistic variable & Fuzzy set \\
\hline ECG & $\{$ Low, Normal, High $\}$ \\
\hline BP & $\{$ Low, Normal, High $\}$ \\
\hline FBS & $\{$ Low, Normal, High $\}$ \\
\hline Cholesterol & $\{$ Low, Normal, High $\}$ \\
\hline Detection & $\{$ No, Yes $\}$ \\
\hline
\end{tabular}

The membership functions input ECGRange

Low

Normal

0

Abnormal

The membership functions input BP Range

Low

Normal

High

[70/110-80/120]

[90/130 and above]

The membership functions input FBS $\underline{\text { Range }}$

$\begin{array}{ll}\text { Low } & {[60-70]} \\ \text { Normal } & {[70-120]} \\ \text { High } & {[120 \text { and above }]}\end{array}$

The membership functions input Cholesterol Range

\begin{tabular}{ll}
\hline Low & {$[130]$} \\
Normal & {$[130-230]$} \\
High & {$[230$ and above] }
\end{tabular}

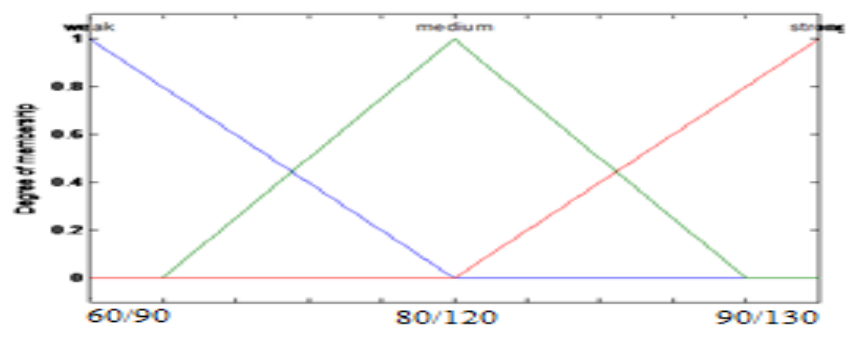

BP

Fig. 8 Membership Function of input BP

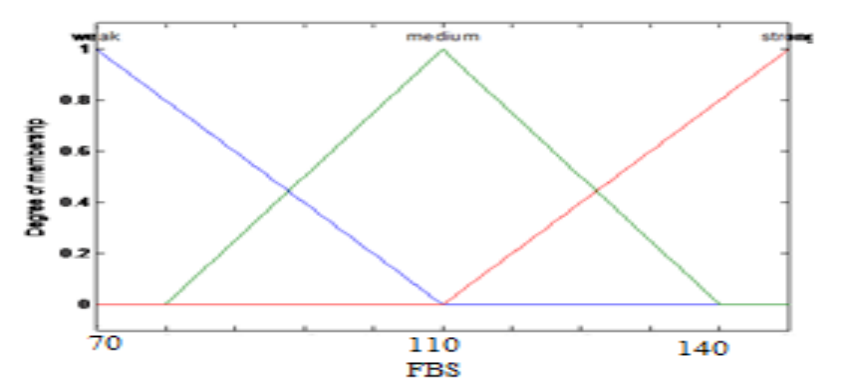

Fig. 9 Membership Function of input FBS
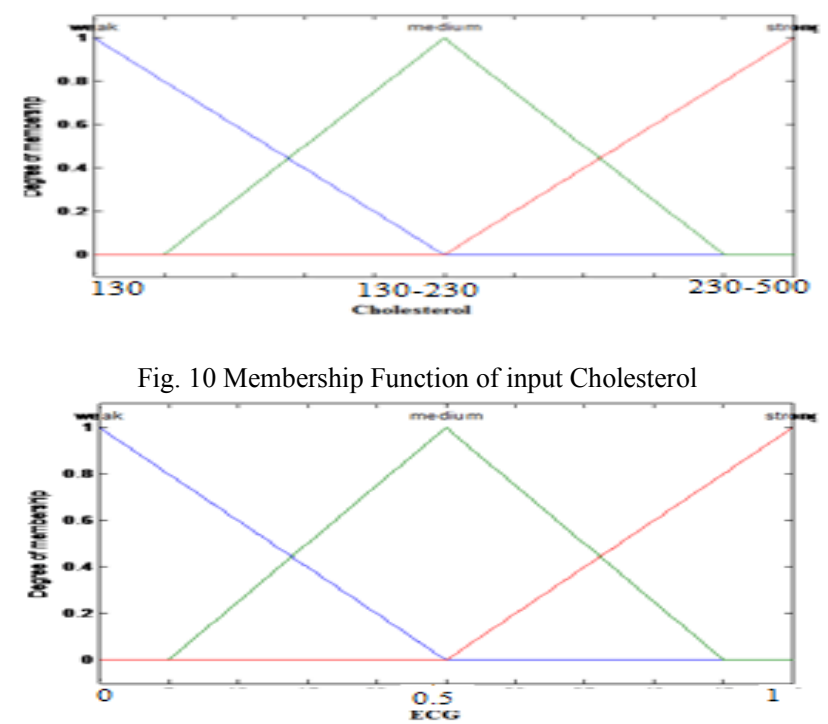

Fig. 11 Membership Function of input ECG

Once the detection is performed it has to be communicated to different agents. The communication module may consist of either internet technology or the mobile network or of the both through the smart devices. The digital mobile communication systemis widely useddue to its high reliability.SMS (Short Message Service)as it is accessible by everyone those having mobiles. Also it is possible to have internet in smart mobile system as well as the computer terminal. In case of computer terminal a modem is required as an additional device. In this case it has been verified for both and the result shown in the result section.

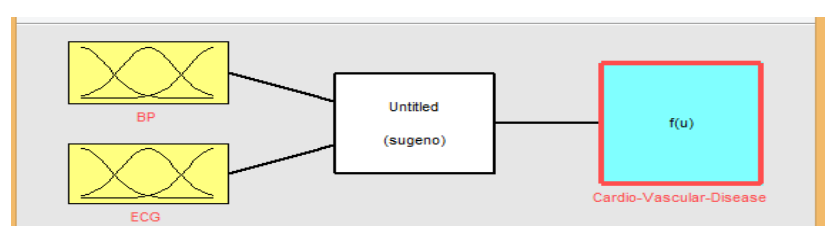

Fig. 12 FIS editor window showing the inputs and outputs

The FIS editor window shown the inputs and outputs has been shown in Fig. 12. The fuzzy rule viewer for BP and ECG for detection of Cardio-Vascular disease is shown in Fig. 13 and the rule formation in Fig. 14. 

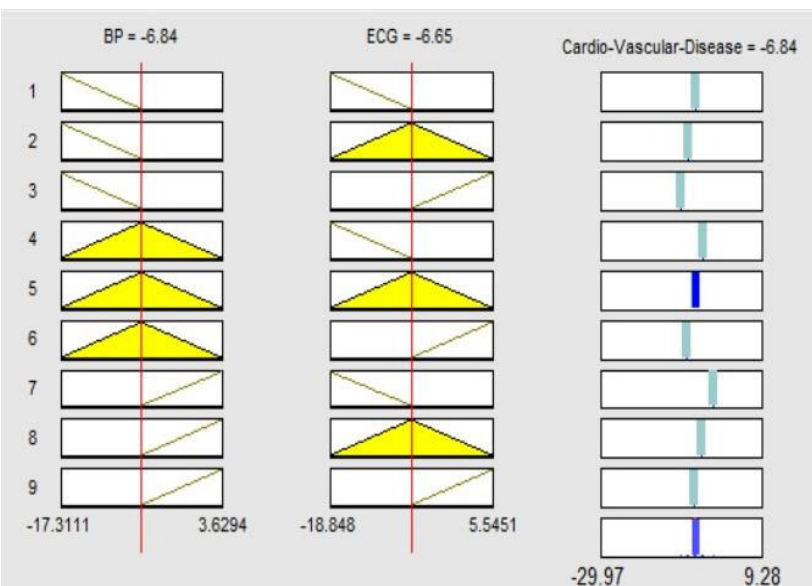

Fig. 13 Fuzzy rule viewer for BP and ECG for detection of Cardio-Vascular disease

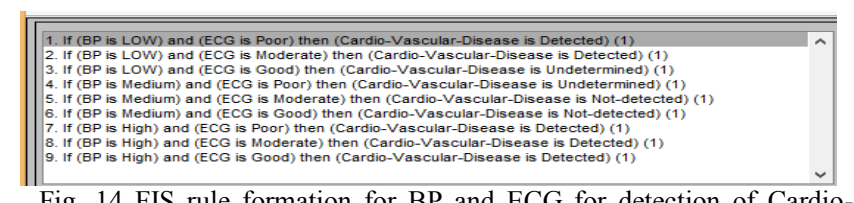
Vascular disease

\section{RESULTS}

The accessed data is represented in table- $\mathrm{V}$ and popup menu at the time of sending and receiving mail is shown in Fig. 16. From this report it can be diagnosed. As the data shown it appears to be the patient is suffering from cardiac problem. Table V

\begin{tabular}{|l|l|l|}
\hline BP & Cholesterol & FBS \\
\hline $95 / 160$ & 400 & 250 \\
\hline $90 / 140$ & 350 & 190 \\
\hline
\end{tabular}

Similarly the snapshot of accessed data in MATLAB environment is shown in Fig. 15.

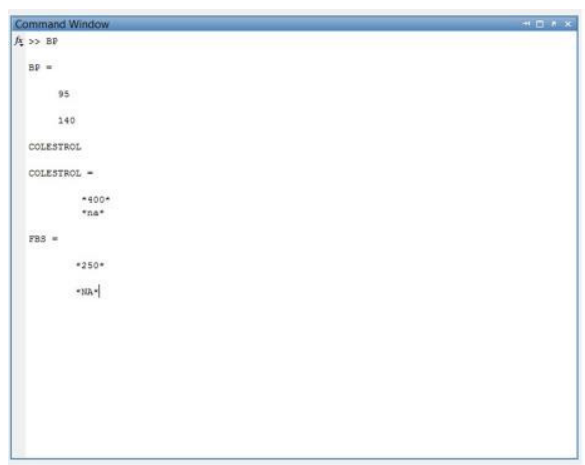

Fig.15Snapshot of Data in MATLAB Environment

Further the data can be sent/received through mail service using popup menu as shown in Fig. 16. The report is sent through the mobile message. The Popup menu shows the structure of FIS.

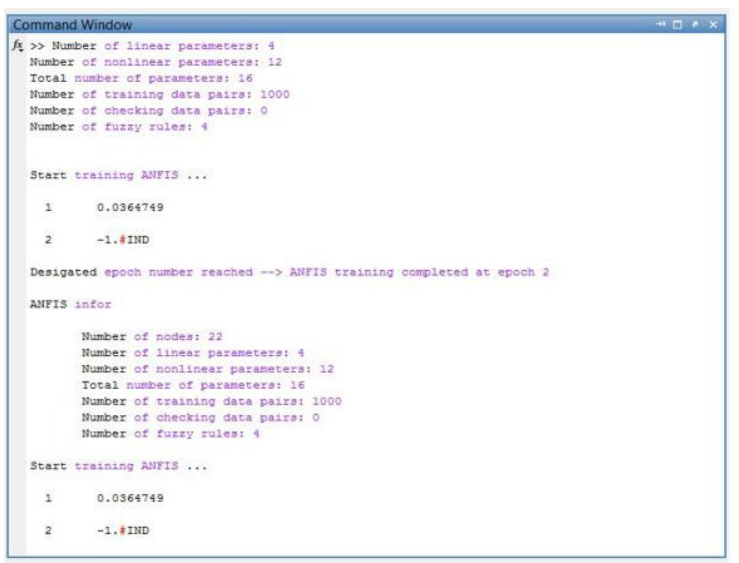

Fig. 16Snapshot for Popup menu at the time of sending and receiving the mail

Similarly the SMS service using GSM module is represented in Fig.17 as follows. Depending upon the various parameters sent by the patient it is detected that the patient is normal. So status of the patient is intimated as OK.

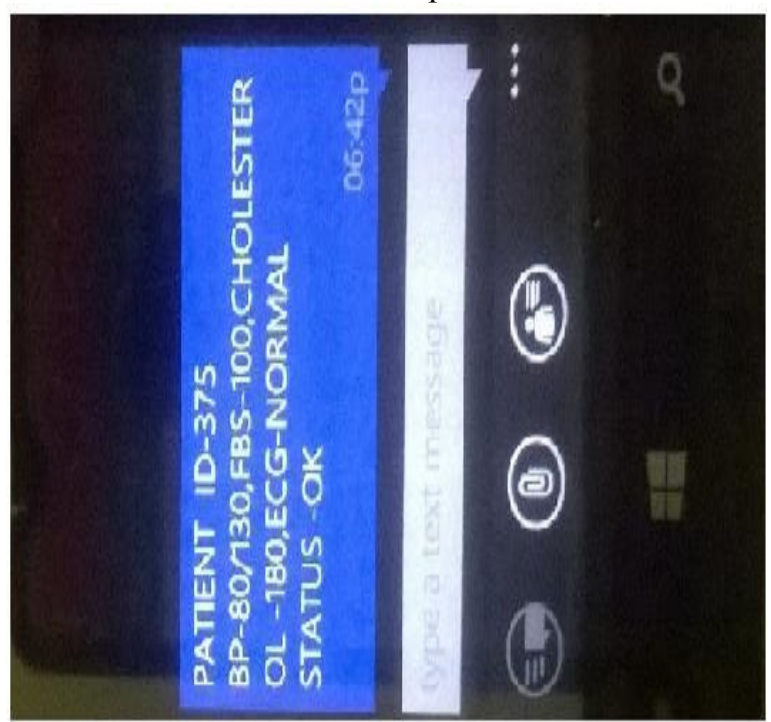

Fig. 17 Snapshot of SMS received from the server

\section{CONCLUSION}

We have proposed this model for disease detection and monitoring patients at remote locations. The distributed agents are proposed to act on a common platform using internet for the benefit of the society. The system database store all the attributes for future reference and implementation of Fuzzy Inference system for diagnosis saves the time of physicians as well as save the travelling cost of the patient. This ingenious idea can be implemented to other crucial areas for future work like disaster management, green house monitoring, food and agriculture industry, etc.

\section{REFERENCES}

[1] H.D Lee, A.Rabbi, J.Choi, and R.F,Rezai, "Development of a Mobile Phone Based e-Health Monitoring 
Application," International Journal of Advanced Computer Science and Applications, vol. 3, no. 3, pp. 38-43,2012.

[2] J. C. Su, "Mobile multi-agent based, distributed information platform (MADIP) for wide-area e-health monitoring", Computers in Industry, vol. 59, pp: 55-68, 2008.

[3] N. Benhajiji, D.Roy, and D. Anciaux, "Patient-centered multi agent system for health care",IFAC-Papers On Line, vol. 48, no. 3, pp: 710714, 2015.

[4] G. B. Silverman, N. Hanrahan, G. Bharathy, K. Gordon K, and D. Johnson, "A systems approach to healthcare: Agent-based modeling,community mental health, and population well-being", Artificial Intelligence in Medicine,vol. 63, pp. 61-71, 2015.

[5] H. O. Al-Sakran, "Framework architecture for improving healthcare information systems using agent technology", International Journal of Managing Information Technology,vol.7, no.1, pp. 1731,February2015

[6] K. AlSharqi, A. Abdelbari,,A. Abou-Elnour, and M. Tarique M, "Zigbee based wearable remote healthcare monitoring system for elderly patients" International Journal of Wireless \& Mobile Networks (IJWMN), 2014; June, Vol. 6, No. 3, pp-53-67.

[7] M. N. Mohanty, "An Efficient Design for Low Cost Cardio-Monitoring System", Adv. Science Letters, vol.22, Iss. 2, pp. 349-353,2016

[8] S. S. Biswal, M. N. Mohanty, B. Sahu, and S. Das, "Unconscious State Analysis Using Intelligent Signal Processing Techniques", Adv. Science Letters, vol.22, Iss. 2, pp. 314-318,2016

[9] A. Das, S. S. Biswal,S. Shalinee, and M. N. Mohanty, "Design of Telemedicine System for Brain Signal Analysis", IJTMCP, Inder Sc. Pub., vol.1, no. 3,2016

[10] M. Singh, M. N. Mohanty, and R. N. D. Choudhury, "An Embedded Design for Patient Monitoring and Telemedicine",Int.Journal of Electrical, Electronics \& Computer Engineering", vol. 2, no. 2, pp. 66-71, 2013

[11] C. S. Devi, G. G. Ramani,and J. A. Pandian, "Intelligent E-Healthcare Management System in Medicinal Science", International Journal of PharmTech Research, vol. 6, no.6, pp. 1838-1845,Oct-Nov. 2014.

[12] E. E. Elavathingal, and T.K Sethuramalingam, "A Survey of EHealthcare Systems Using Wearable Devices and Medical Sensor Networks", Karpagam journal of engineering research (KJER),vol1,no.1Iss. 1,2014

[13] S. Tupe, and N. P. Kulkarni, "ECA: Evolutionary Computing Algorithm for E-Healthcare Information System", SPPU, Pune iPGCON-2015.

[14] S. A. Hannan, A. V. Mane, R. R. Manza, and R. J. Ramteke, "Prediction of heart disease medical prescription using radial basis function",978-1-4244-5967-4/10/\$26.00 C IEEE, 2010.
[15] M. Shouman, T. Turner, and R. Stocker, "Using Decision Tree for Diagnosing Heart Disease Patients",Conferences in Research and Practice in Information Technology (CRPIT),vol. 121, pp. 23-29,2011.

[16] M. Shouman, T. Turner, and R. Stocker, "Applying k-Nearest Neighbour in Diagnosing Heart Disease Patients",International Journal of Information and Education Technology,vol. 2, no. 3, pp. 220223, June 2012.

[17] D. Mandal, I. M. Chattopadhyay, and S. Mishra, "A Low Cost Noninvasive Digital Signal Processor Based (TMS320C6713) Heart Diagnosis System", 1st Int'l Conf. on Recent Advances in Information Technology, RAIT-2012.

[18] B. Venkatalakshmi, and M.V. Shivsankar, "Heart Disease Diagnosis Using Predictive Data mining"International Journal of Innovative Research in Science, Engineering and Technology,vol. 3, Special Iss. 3, pp. 1873-1877,March2014.

[19] Z. F. Fitrilina, and H. I. K. Kamil, "Prototype Early Warning System for Heart Disease Detection UsingAndroid Application”, 978-1-42447929-0/14/\$26.00 @2014, IEEE, pp. 3468-3471, 2014.

[20] Q. A. Rahman, L. G. Tereshchenko, M. Kongkatong, T. M. Abraham, M. R. Abraham, and H. Shatkay, "Utilizing ECG-Based Heartbeat Classification for Hypertrophic Cardiomyopathy Identification", IEEE transactions on nanobioscience, vol. 14, no. 5, pp. 505-512, July 2015.

[21] A. Forkan, I. Khalil, T. Z. Zahir, "Context-aware Cardiac Monitoring for Early Detection of Heart Diseases", Computing in Cardiology; vol. 40, pp.277-280, 2013.

[22] L. Sarangi, M. N. Mohanty and S. Patnaik "Design of MLP Based Model for Analysis of Patient Suffering from Influenza" Proceedia Computer Science, Elsevier, 92, 2016; pp-396-403.

[23] L. Sarangi, M. N. Mohanty and S. Patnaik "An Intelligent Decision Support System for Cardiac Disease Detection" International Journal of Control Theory and Applications, 8(5), 2015; pp. 2137-2143.

[24] L. Sarangi, M. N. Mohanty and S. Patnaik "Critical Heart Condition Analysis through Diagnostic Agent of e-Healthcare System using Spectral Domain Transfom" Indian Journal of Science \& Technology, Vol 9(38) 2016; pp. 1-6

[25] Semwal, Vijay Bhaskar, Pavan Chakraborty, and Gora Chand Nandi. "Less computationally intensive fuzzy logic (type-1)-based controller for humanoid push recovery." Robotics and Autonomous Systems 63 (2015): 122-135.

[26] Kumari, Pinki, and Abhishek Vaish. "Information-theoretic measures on intrinsic mode function for the individual identification using EEG sensors." IEEE Sensors Journal 15.9 (2015): 4950-4960.

[27] Kumari, Pinki, and Abhishek Vaish. "Feature-level fusion of mental task's brain signal for an efficient identification system." Neural Computing and Applications 27.3 (2016): 659-669. 